\title{
A seakeeping analysis method for an air-lifted vessel
}

\author{
Nan Xie, D. Vassalos, A. Jasionowski, P. Sayer* \\ Department of Naval Architecture and Marine Engineering, Universities of Glasgow and Strathclyde, Henry Dyer Building, 100 Montrose Street, Glasgow G4 0LZ, UK
}

\section{A R T I C L E I N F O}

\section{Article history:}

Received 14 October 2007

Accepted 12 June 2008

Keywords:

Seakeeping

Frequency domain

Air-lifted vessel

Numerical prediction

\begin{abstract}
A B S T R A C T
A seakeeping analysis in the frequency domain is presented to predict the motion response of an airlifted vessel (ALV) in waves. The ALV is supported by pressurised air in two separate cushion chambers; the pressure variation in the cushions has a significant effect on the motions of the vessel. The adiabatic gas law is used to couple cushion pressure and the free-surface elevation of water inside the chamber. Attention is focused on the waves generated by the pressure, and a method is presented to compute the corresponding free-surface elevation. New numerical schemes are proposed for calculating the threedimensional free-surface elevation for the four wave numbers. Numerical results of the free-surface elevation, escape area, escape volume and motion responses of the ALV are provided.
\end{abstract}

(c) 2008 Elsevier Ltd. All rights reserved.

\section{Introduction}

The innovative design of an air-lifted vessel (ALV) utilises the cushioning and lubricating properties of air to generate an efficient high-speed marine vehicle. An energy saving of up to $25 \%$ is possible compared to conventional high-speed mono-hulls or catamarans, based on model experiments by Allenstrom et al. (2001, 2003).

The selected ALV is a skirtless SES catamaran. There are two demi-hulls, each of which contain an air cushion (see Fig. 1). The design feature of the ALV is similar, in some sense, to that of an SES catamaran. There are two demi-hulls, each of which contains air cushions. A forward planing surface ahead of the step creates some limited dynamic lift. The step and the forward planing surface also create the forward ceiling of the air cushion chamber, the step being located in a plane close to horizontal (no part of the step is to ventilate before the other). The bow section is slender in order to reduce displacement forces in a seaway, and the bow incorporate a voluminous part above a built-in chine/spray rail to reduce water deflection and reserve buoyancy in pitching motion. The side keels of varying height extend from just ahead of the step to the transom. The height of the keels is adjusted to the observed/expected shape of the air cushion. The only purpose of the side keels is to fence in the air cushion. There are spray rails on the outside of the side keels, at a height partly to deflect the water without wetting the rails on the upper sides. The cushion ceiling is at a height to avoid being hit by waves when the vessel is moving. The ceiling aft end slopes to deflect passing waves. A flap or enclosure arrangement in line with the sloped portion of the ceiling is positioned to 'fence' the air cushion chamber in the rear part and to create dynamic lift and motion damping. The air

\footnotetext{
* Corresponding author. Tel.: +44141548 3302; fax: +44141552 2879.

E-mail address: p.g.sayer@na-me.ac.uk (P. Sayer).
}

cushion concept of the present ALV is patented (EFFISES Project, 2001-2004). More details about the ALV concept can be found in Allenstrom et al. (2001).

In the 'on-cushion' mode, the vessel is mainly supported by the difference between the ambient atmospheric pressure and that in the plenum chamber. The pressure of the air captured in the chamber undergoes a pressure variation, and thus waves are generated on the free-surface. The dynamic response of the ALV in turn causes a reaction on the craft through the captured air, which generates further variation of the air pressure. The motions affect passenger and crew comfort as well as the performance of electronic components and machinery on board. In the present paper, a method is presented for the numerical prediction of the seakeeping performance of the ALV; this is similar to that for a surface-effect ship (SES), previously studied by other researchers, including Doctors (1976), Chen (1977), Kim and Tsakonas (1981), Kaplan et al. (1981), and Faltinsen (2005).

As the ALV is supported by pressurised air in two separate cushion chambers, the behaviour of the craft in waves is mainly due to the pressure variation in the cushion chambers. The adiabatic gas law is used to couple cushion pressure and the freesurface elevation inside the chambers. Computations of the corresponding wave patterns are presented. Efficient and effective numerical schemes are proposed for calculating the threedimensional free-surface elevation for the four wave numbers. Numerical results are also presented for the free-surface elevation, air cushion escape area and escape volume, and for the motion of the vessel in irregular waves.

\section{Equations of motion of the ALV}

A right-handed coordinate system oxyz is adopted with the plane $z=0$ in the undisturbed free-surface and $z$ positive 


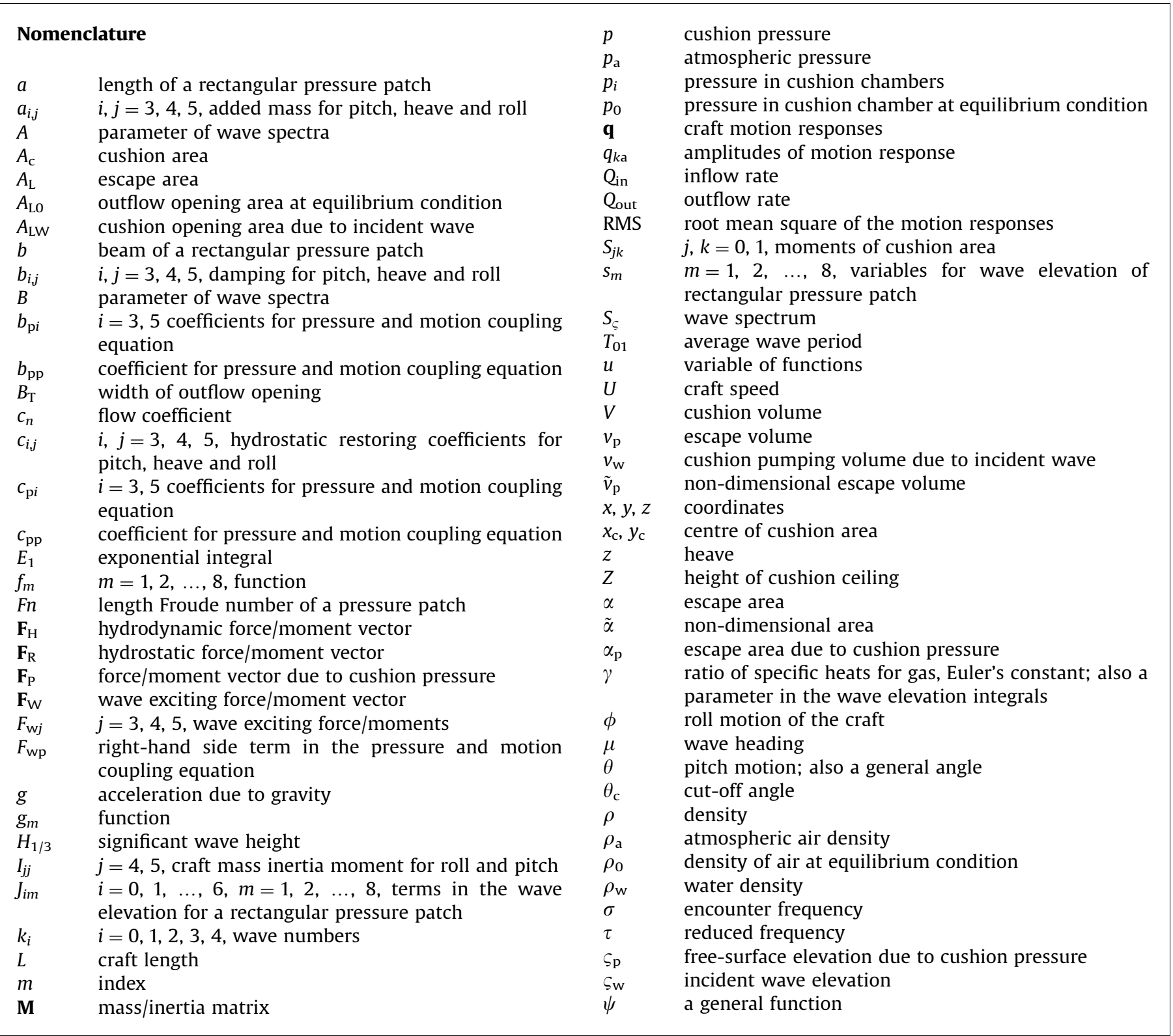

upwards. The origin is located at C.G. of the vessel. Frequency domain analysis method will be used in the present study. The equation of motions of the ALV can be expressed as

$[\mathbf{M}][\ddot{\mathbf{q}}]=\left[\mathbf{F}_{\mathrm{H}}\right]+\left[\mathbf{F}_{\mathrm{R}}\right]+\left[\mathbf{F}_{\mathrm{P}}\right]+\left[\mathbf{F}_{\mathrm{W}}\right]$,

where $\mathbf{q}$ is the motion vector of the vessel, $\mathbf{M}$ is the mass/inertia matrix, $\mathbf{F}_{\mathrm{H}}$ is the hydrodynamic force/moment, $\mathbf{F}_{\mathrm{R}}$ is the hydrostatic force/moment, $\mathbf{F}_{\mathrm{P}}$ is the force/moment due to the cushion pressure, and $\mathbf{F}_{\mathrm{W}}$ is the wave exciting force/moment.

The adiabatic gas law and continuity equation for the air in each chamber are used to determine the cushion pressure in the port and starboard chambers:

$\left\{\begin{array}{l}\frac{p_{i}+p_{\mathrm{a}}+p_{0, i}}{\rho_{i}^{\gamma}}=\text { constant, } \\ V_{i} \frac{\mathrm{d} \rho_{i}}{\mathrm{~d} t}+\rho_{i} \frac{\mathrm{d} V_{i}}{\mathrm{~d} t}=\rho_{\mathrm{a}} Q_{\mathrm{in}, i}-\rho_{\mathrm{a}} Q_{\text {out }, i},\end{array}\right.$

where the subscripts $i(=1,2)$ denote the port and starboard cushions, respectively; henceforth, for clarity this subscript will be omitted unless specifically required; $p_{0}$ and $\rho_{0}$ are cushion pressure and air density at equilibrium operating condition; $p_{\mathrm{a}}$ and $\rho_{\mathrm{a}}$ are atmospheric pressure and air density; $Q_{\text {in }}$ and $Q_{\text {out }}$ are the inflow and out flow rate; $V$ is the cushion volume, determined by vessel motion attitude, incident wave and cushion pressure:

$V=\iint_{A_{c}}\left[Z(x, y)-\varsigma_{\mathrm{p}}(x, y)-\varsigma_{\mathrm{w}}(x, y)\right] \mathrm{d} x \mathrm{~d} y$

where $Z$ is the cushion ceiling height, $\varsigma_{\mathrm{w}}$ and $\varsigma_{\mathrm{p}}$ are incident wave and free-surface elevation due to cushion pressure, respectively. The outflow rate can be expressed as

$Q_{\text {out }}=c_{n} A_{\mathrm{L}} \sqrt{\frac{2\left(p+p_{0}\right)}{\rho_{\mathrm{a}}}}$

where $c_{n}(\approx 0.6-0.8)$ is the flow coefficient and $A_{\mathrm{L}}$ is the air escape area of the flow opening at the cushion stern boundary:

$A_{\mathrm{L}}=\int_{y_{\mathrm{a}}}^{y_{\mathrm{b}}}\left[Z\left(x_{\mathrm{T}}, y\right)-\varsigma_{\mathrm{p}}\left(x_{\mathrm{T}}, y\right)-\varsigma_{\mathrm{w}}\left(x_{\mathrm{T}}, y\right)\right] \mathrm{d} y$

where $x_{\mathrm{T}}$ is the longitudinal coordinate of cushion stern aperture. 


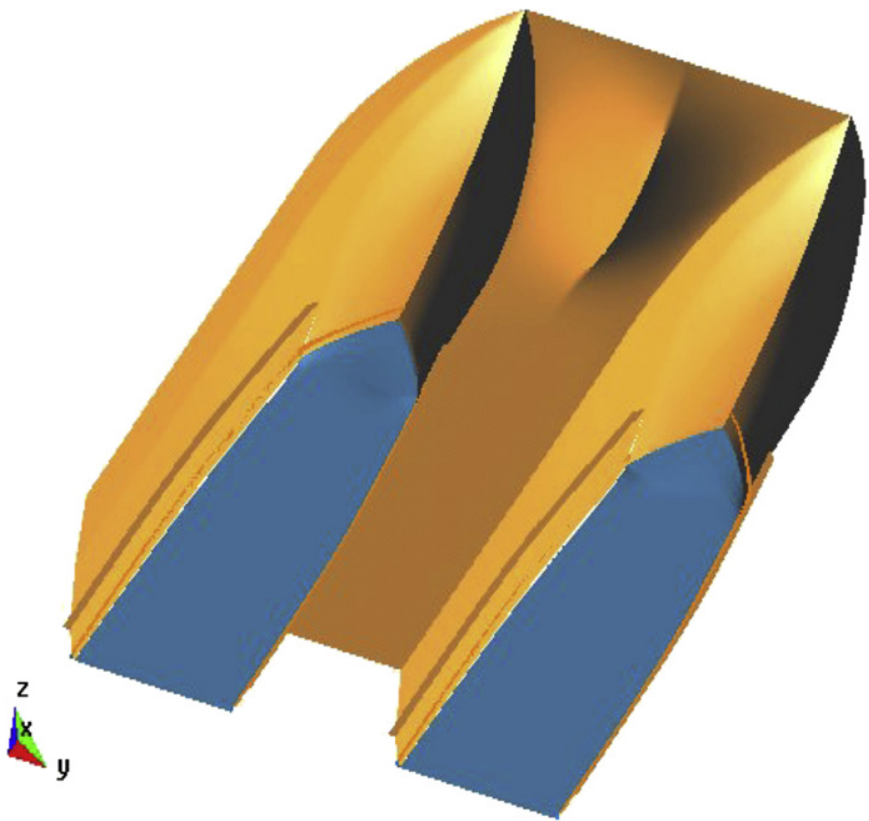

Fig. 1. The ALV concept.

Expanding (2) about the vessel's equilibrium position, and assuming small amplitudes of motion for the ALV, the linearised cushion pressure equation can be written as

$b_{\mathrm{pp}} \dot{p}+c_{\mathrm{pp}} p+b_{\mathrm{p} 3} \dot{z}+c_{\mathrm{p} 3} z+b_{\mathrm{p} 5} \dot{\theta}+c_{\mathrm{p} 5} \theta+b_{\mathrm{p} 4} \dot{\phi}+c_{\mathrm{p} 4} \phi=F_{\mathrm{wp}}$

where

$b_{\mathrm{pp}}=\frac{V_{0} \rho_{0}^{1+\gamma}}{\gamma p_{0}}-\rho_{0} v_{\mathrm{p}}$

$c_{\mathrm{pp}}=\rho_{\mathrm{a}}\left|\frac{\partial Q_{\mathrm{in}}}{\partial p}\right|_{0}+c_{n} \frac{\rho_{\mathrm{a}} A_{\mathrm{L} 0}}{\sqrt{2 p_{0} \rho_{\mathrm{a}}}}-c_{n} \alpha \sqrt{\frac{2 p_{0}}{\rho_{\mathrm{a}}}}$

$b_{\mathrm{p} 3}=-\rho_{0} A_{\mathrm{c}}$

$c_{\mathrm{p} 3}=-c_{n} \rho_{\mathrm{a}} B_{\mathrm{T}} \sqrt{\frac{2 p_{0}}{\rho_{\mathrm{a}}}}$

$b_{\mathrm{p} 5}=\rho_{0} x_{\mathrm{c}} A_{\mathrm{c}}$

$c_{\mathrm{p} 5}=c_{n} \rho_{\mathrm{a}} x_{\mathrm{c}} B_{\mathrm{T}} \sqrt{\frac{2 p_{0}}{\rho_{\mathrm{a}}}}$

$b_{\mathrm{p} 4}=-\rho_{0} y_{\mathrm{c}} A_{\mathrm{c}}$

$c_{\mathrm{p} 4}=-c_{n} \rho_{\mathrm{a}} y_{\mathrm{c}} B_{\mathrm{T}} \sqrt{\frac{2 p_{0}}{\rho_{\mathrm{a}}}}$

$F_{\mathrm{wp}}=\rho_{0} \dot{v}_{\mathrm{w}}+c_{n} A_{\mathrm{Lw}} \sqrt{\frac{2 p_{0}}{\rho_{\mathrm{a}}}}$

In the above, $V_{0}$ is the cushion volume at equilibrium condition; $\left(x_{\mathrm{c}}, y_{\mathrm{c}}\right)$ is the cushion centre; $A_{\mathrm{c}}$ is the cushion area; $\partial Q_{\mathrm{in}} / \partial_{\mathrm{p}}$ is the discharge by fan per unit pressure; $v_{\mathrm{p}}$ and $\alpha_{\mathrm{p}}$ are the escape volume and escape area resulting from unit cushion pressure; $z, \theta$, $\phi$ are heave, pitch and roll motions; $A_{\mathrm{LO}}$ is the outflow opening area at equilibrium condition; $B_{\mathrm{T}}$ is the width of the outflow opening; $v_{\mathrm{w}}$ and $A_{\mathrm{Lw}}$ are the cushion pumping volume and opening area, respectively, due to the incident waves:

$v_{\mathrm{w}}=\iint_{A_{\mathrm{c}}} \varsigma_{\mathrm{w}}(x, y) \mathrm{d} x \mathrm{~d} y$

$A_{\mathrm{LW}}=\int_{y_{\mathrm{a}}}^{y_{\mathrm{b}}} \varsigma_{\mathrm{w}}\left(x_{\mathrm{T}}, y\right) \mathrm{d} y$

The cushion escape volume and escape area are given, respectively, by

$v_{\mathrm{p}}=\iint_{A_{\mathrm{C}}} \varsigma_{\mathrm{p}}(x, y ; \sigma) \mathrm{d} x \mathrm{~d} y$

$\alpha_{\mathrm{p}}=\int_{y_{\mathrm{a}}}^{y_{\mathrm{b}}} \varsigma_{\mathrm{p}}\left(x_{\mathrm{T}}, y ; \sigma\right) \mathrm{d} y$

Physically, the cushion escape volume is the volume resulting from the free-surface elevation, due to cushion pressure, inside the cushion chamber of the ALV; while the escape area is the area generated by this free-surface profile along the stern opening of the cushion chamber. The equations of heave, pitch and roll may be linearised relative to the equilibrium position of the ALV:

$\left\{\begin{array}{l}\left(m+a_{33}\right) \ddot{z}_{3}+b_{33} \dot{z}+c_{33} z+a_{35} \ddot{\theta}+b_{35} \dot{\theta}+c_{35} \theta-S_{00}\left(p_{1}+p_{2}\right)=F_{\mathrm{w} 3}, \\ a_{53} \ddot{z}+b_{53} \dot{z}+c_{53} z+\left(I_{55}+a_{55}\right) \ddot{\theta}+b_{55} \dot{\theta}+c_{55} \theta+S_{10}\left(p_{1}+p_{2}\right)=F_{\mathrm{w} 5}, \\ \left(I_{44}+a_{44}\right) \ddot{\phi}+b_{44} \dot{\phi}+c_{44} \phi-S_{01}\left(p_{1}-p_{2}\right)=F_{\mathrm{w} 4},\end{array}\right.$

where $a_{i j}$ and $b_{i j}$ are the added mass and damping coefficients of the rigid side-hull, $c_{i j}$ is the hydrostatic restoring coefficient, $F_{\mathrm{w} j}$ is the wave exciting force/moment, and

$S_{j k}=\iint_{A_{c}} x^{j} y^{k} \mathrm{~d} x \mathrm{~d} y \quad j, k=0,1$

are the moments of the cushion area. The hydrodynamic coefficients and wave exciting force/moments on the thin rigid side-hull of each cushion are calculated by strip theory.

Hence, the transfer functions for heave, pitch and roll in regular waves can be obtained. The root mean square (RMS) values are

$\operatorname{RMS}_{k}(\mu)=\left(\int_{0}^{\infty}\left(\frac{q_{k \mathrm{a}}(\mu)}{S_{\mathrm{a}}}\right)^{2} S_{\varsigma}(\omega) \mathrm{d} \omega\right)^{1 / 2} k=3,4,5$,

where $\mu$ is wave heading ( $180^{\circ}$ for head waves; $0^{\circ}$ for following waves). The ITTC wave spectrum was used in the present study:

$S_{\varsigma}(\omega)=A \omega^{-5} \exp \left(\frac{-B}{\omega^{4}}\right)$

where $A=173.18 H_{1 / 3}^{2} / T_{01}^{4}$ and $B=692.73 / T_{01}^{4}$ with $H_{1 / 3}$ significant wave height $(\mathrm{m})$ and $T_{01}$ average wave period (s).

For the calculation of the transfer function of the free-surface elevation due to cushion pressure, the cushion areas are represented by a number of rectangular pressure patches, as in Fig. 2.

The major part of the computation is the calculation of the escape volume in the cushion and the escape area at the leakage openings; this is discussed in Section 3.

\section{Free-surface elevation due to oscillatory cushion pressure}

In this section, a method is outlined for calculating the freesurface elevation caused by the ALV cushion pressure. The transfer function of the escape area and escape volume in the cushion will be calculated in the frequency domain. The cushion area is 

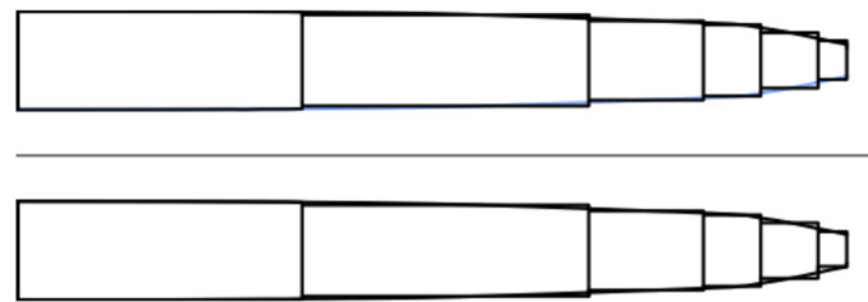

Fig. 2. Representation of the cushion areas of the ALV.

modelled by rectangular pressure patches. The solution for a rectangular pressure patch provides the elementary solution of the problem. The hydrodynamic boundary value problem for a pressure patch oscillating with constant amplitude and moving on the free-surface of a deep, incompressible, ideal fluid was calculated using the corresponding velocity potential. The freesurface elevation due to a moving oscillating rectangular pressure patch with unit pressure strength can be written as, see, for example, Wehausen and Laitone (1960), Kim and Tsakonas (1981), and Xie et al. (2005):

$$
\begin{aligned}
\varsigma_{\mathrm{p}}(x, y)= & -\frac{1}{\rho_{\mathrm{w}} g} \\
& -\frac{\sum_{m=1}^{8}(-1)^{m}\left(J_{0 m}+J_{1 m}+J_{2 m}+J_{3 m}+J_{4 m}+J_{5 m}+J_{6 m}\right)}{4 \rho_{\mathrm{w}} g \pi^{2}}
\end{aligned}
$$

Here, $\rho_{\mathrm{w}}$ is the density of water, and

$$
\begin{aligned}
J_{0 m}= & \int_{0}^{\pi / 2} \frac{2}{\sin \theta \cos \theta} \ln \left|s_{m}(\theta)\right| \mathrm{d} \theta \\
J_{1 m}= & \int_{0}^{\pi / 2} \frac{\left[E_{1}\left(\mathrm{i} k_{1} s_{m}\right)-\pi \mathrm{i}\left(1-\operatorname{sgn}\left(s_{m}\right)\right)\right] \mathrm{e}^{\mathrm{i} k_{1} s_{m}}}{\sin \theta \cos \theta \sqrt{1+4 \tau \cos \theta}} \mathrm{d} \theta \\
J_{2 m}= & -\int_{0}^{\pi / 2} \frac{\left[E_{1}\left(\mathrm{i} k_{2} s_{m}\right)-\pi \mathrm{i}\left(1-\operatorname{sgn}\left(s_{m}\right)\right)\right] \mathrm{e}^{\mathrm{i} k_{2} s_{m}}}{\sin \theta \cos \theta \sqrt{1+4 \tau \cos \theta}} \mathrm{d} \theta \\
J_{3 m}= & \int_{\theta_{\mathrm{c}}}^{\pi / 2} \frac{E_{1}\left(-\mathrm{i} k_{3} s_{m}\right) \mathrm{e}^{-\mathrm{i} k_{3} s_{m}}}{\sin \theta \cos \theta \sqrt{1-4 \tau \cos \theta} \mathrm{d} \theta} \\
& -\pi \int_{\theta_{\mathrm{c}}}^{\pi / 2} \frac{\sin \left(k_{3} s_{m}\right)\left[1+\operatorname{sgn}\left(s_{m}\right)\right]}{\sin \theta \cos \theta \sqrt{1-4 \tau \cos \theta}} \mathrm{d} \theta \\
& -\mathrm{i} \pi \int_{\theta_{\mathrm{c}}}^{\pi / 2} \frac{\cos \left(k_{3} s_{m}\right)\left[1+\operatorname{sgn}\left(s_{m}\right)\right]}{\sin \theta \cos \theta \sqrt{1-4 \tau \cos \theta}} \mathrm{d} \theta
\end{aligned}
$$

$$
\begin{aligned}
J_{4 m}= & -\int_{\theta_{\mathrm{c}}}^{\pi / 2} \frac{E_{1}\left(-\mathrm{i} k_{4} s_{m}\right) \mathrm{e}^{-\mathrm{i} k_{4} s_{m}}}{\sin \theta \cos \theta \sqrt{1-4 \tau \cos \theta}} \mathrm{d} \theta \\
& -\pi \int_{\theta_{\mathrm{c}}}^{\pi / 2} \frac{\sin \left(k_{4} s_{m}\right)\left[1-\operatorname{sgn}\left(s_{m}\right)\right]}{\sin \theta \cos \theta \sqrt{1-4 \tau \cos \theta}} \mathrm{d} \theta \\
& -\mathrm{i} \pi \int_{\theta_{\mathrm{c}}}^{\pi / 2} \frac{\cos \left(k_{4} s_{m}\right)\left[1-\operatorname{sgn}\left(s_{m}\right)\right]}{\sin \theta \cos \theta \sqrt{1-4 \tau \cos \theta}} \mathrm{d} \theta
\end{aligned}
$$

$$
\begin{aligned}
J_{5 m}= & -\mathrm{i} \int_{0}^{\theta_{\mathrm{c}}} \frac{E_{1}\left(-\mathrm{i} k_{3} s_{m}\right) \mathrm{e}^{-\mathrm{i} k_{3} s_{m}}}{\sin \theta \cos \theta \sqrt{4 \tau \cos \theta-1}} \mathrm{~d} \theta \\
& -\frac{\pi}{2} \int_{0}^{\theta_{\mathrm{c}}} \frac{\mathrm{e}^{k_{3 i} s_{m}} \cos \left(k_{3 r} s_{m}\right)\left[1+\operatorname{sgn}\left(s_{m}\right)\right]}{\sin \theta \cos \theta \sqrt{4 \tau \cos \theta-1}}\left[1+\operatorname{sgn}\left(k_{3 r}\right)\right] \mathrm{d} \theta \\
& +\frac{\mathrm{i} \pi}{2} \int_{0}^{\theta_{\mathrm{c}}} \frac{\mathrm{e}^{k_{3 i} s_{m}} \sin \left(k_{3 r} s_{m}\right)\left[1+\operatorname{sgn}\left(s_{m}\right)\right]}{\sin \theta \cos \theta \sqrt{4 \tau \cos \theta-1}}\left[1+\operatorname{sgn}\left(k_{3 r}\right)\right] \mathrm{d} \theta
\end{aligned}
$$

$$
\begin{aligned}
J_{6 m}= & \mathrm{i} \int_{0}^{\theta_{c}} \frac{E_{1}\left(-\mathrm{i} k_{4} s_{m}\right) \mathrm{e}^{-\mathrm{i} k_{4} s_{m}}}{\sin \theta \cos \theta \sqrt{4 \tau \cos \theta-1}} \mathrm{~d} \theta \\
& -\frac{\pi}{2} \int_{0}^{\theta_{\mathrm{c}}} \frac{\mathrm{e}^{k_{4 i} s_{m}} \cos \left(k_{4 r} s_{m}\right)\left[1-\operatorname{sgn}\left(s_{m}\right)\right]}{\sin \theta \cos \theta \sqrt{4 \tau \cos \theta-1}}\left[1+\operatorname{sgn}\left(k_{4 r}\right)\right] \mathrm{d} \theta \\
& +\frac{\mathrm{i} \pi}{2} \int_{0}^{\theta_{\mathrm{c}}} \frac{\mathrm{e}^{k_{4 i} s_{m}} \sin \left(k_{4 r} s_{m}\right)\left[1-\operatorname{sgn}\left(s_{m}\right)\right]}{\sin \theta \cos \theta \sqrt{4 \tau \cos \theta-1}}\left[1+\operatorname{sgn}\left(k_{4 r}\right)\right] \mathrm{d} \theta
\end{aligned}
$$

Furthermore, $E_{1}\left[-\mathrm{i} k_{1} s_{m}\right]$ is the exponential integral of complex argument (Abramowitz and Stegun, 1970):

$$
E_{1}(z)=\int_{z}^{\infty} \frac{\mathrm{e}^{-u}}{u} \mathrm{~d} u=-\gamma-\log z-\sum_{n=1}^{\infty} \frac{(-1)^{n} z^{n}}{n \times n !}
$$

where $\gamma=0.5772 \ldots$ is Euler's constant.

Finally,

$\theta_{c}= \begin{cases}\operatorname{tg}^{-1}\left(\frac{1}{4 \tau}\right), & \tau>0.25 \\ 0, & \tau<0.25\end{cases}$

$s_{m}(\theta)= \begin{cases}(x-a) \cos \theta+(y-b) \sin \theta, & m=1 \\ (x-a) \cos \theta+(y+b) \sin \theta, & m=2 \\ (x+a) \cos \theta+(y+b) \sin \theta, & m=3 \\ (x+a) \cos \theta+(y-b) \sin \theta, & m=4 \\ (x-a) \cos \theta-(y+b) \sin \theta, & m=5 \\ (x-a) \cos \theta-(y-b) \sin \theta, & m=6 \\ (x+a) \cos \theta-(y-b) \sin \theta, & m=7 \\ (x+a) \cos \theta-(y+b) \sin \theta, & m=8\end{cases}$

where $a$ and $b$ are half-length and beam of the rectangular. The four wave numbers are

$$
\begin{aligned}
& k_{1}=\frac{1}{2} k_{0} \sec ^{2} \theta[1+2 \tau \cos \theta-\sqrt{1+4 \tau \cos \theta}], \\
& k_{2}=\frac{1}{2} k_{0} \sec ^{2} \theta[1+2 \tau \cos \theta+\sqrt{1+4 \tau \cos \theta}], \\
& k_{3}=\frac{1}{2} k_{0} \sec ^{2} \theta[1-2 \tau \cos \theta-\sqrt{1-4 \tau \cos \theta}], \\
& k_{4}=\frac{1}{2} k_{0} \sec ^{2} \theta[1-2 \tau \cos \theta+\sqrt{1-4 \tau \cos \theta}],
\end{aligned}
$$

where $k_{0}=\left(g / U^{2}\right)$ is wave number, $\tau=(U \sigma / g)$ is the reduced frequency, and $\sigma$ is the encounter frequency.

We also note that:

$$
\begin{aligned}
& \lim _{\theta \rightarrow \pi / 2}\left\{k_{1}\right\}=k_{0} \tau^{2}=\frac{\sigma^{2}}{g}, \quad \lim _{\theta \rightarrow \pi / 2}\left\{k_{2}\right\}=+\infty, \\
& \lim _{\theta \rightarrow \pi / 2}\left\{k_{3}\right\}=k_{0} \tau^{2}=\frac{\sigma^{2}}{g}, \quad \lim _{\theta \rightarrow \pi / 2}\left\{k_{4}\right\}=+\infty .
\end{aligned}
$$

The evaluation of the free-surface elevation (24) is hampered by singularities in the integrand of $J_{i m}$, and by their highly oscillatory behaviour. For example, the integrand of $J_{0 m}$ becomes infinite when $\theta \rightarrow 0$ and $\theta \rightarrow \pi / 2$; also $k_{2} s_{m} \rightarrow \pm \infty$ as $\theta \rightarrow \pi / 2$.

To overcome these difficulties, we write $F_{m}(\theta)=f_{m}(\theta) / g_{m}(\theta)$, where $f_{m}(\theta)$ is regular and $g_{m}(\theta)$ has a zero of order one at $\theta=\theta_{\mathrm{a}}$. Thus, the integral of $F_{m}(\theta)$ may be written as

$$
\begin{aligned}
I_{m}= & \int_{\theta_{\mathrm{a}}}^{\theta_{\mathrm{b}}} \frac{f_{m}(\theta)}{g_{m}(\theta)} \mathrm{d} \theta=\int_{\theta_{\mathrm{a}}}^{\theta_{\mathrm{b}}}\left[\frac{f_{m}(\theta)}{g_{m}(\theta)}-\frac{f_{m}\left(\theta_{\mathrm{a}}\right)}{g_{m}^{\prime}\left(\theta_{\mathrm{a}}\right)\left(\theta-\theta_{\mathrm{a}}\right)}\right] \mathrm{d} \theta \\
& +\int_{\theta_{\mathrm{a}}}^{\theta_{\mathrm{b}}} \frac{f_{m}\left(\theta_{\mathrm{a}}\right)}{g_{m}^{\prime}\left(\theta_{\mathrm{a}}\right)\left(\theta-\theta_{\mathrm{a}}\right)} \mathrm{d} \theta .
\end{aligned}
$$

On the right-hand side of (41), we observe that the integrand of the first integral is regular at $\theta=\theta_{\mathrm{a}}$; furthermore, the second integral makes no contribution to the summation in (24). The same approach can be applied to the case when $\theta=\theta_{\mathrm{b}}$. In this way, the singularities in the integrals of the free-surface elevation may be removed. 
In the case of $J_{3 m}, J_{4 m}, J_{5 m}$ and $J_{6 m}$, there is a singularity of order $1 / 2$ at $\theta=\theta_{\text {c }}$ which is integrable:

$\sqrt{1-4 \tau \cos \theta}=\sqrt{\frac{2}{\tau}} \sqrt{\sin \left(\frac{\theta+\theta_{\mathrm{c}}}{2}\right)} \sqrt{\sin \left(\frac{\theta-\theta_{\mathrm{c}}}{2}\right)}$

when $\theta \in\left(\theta_{\mathrm{c}}, \pi / 2\right)$, and a direct integration scheme can be used to calculate $J_{0 m}, J_{1 m}, J_{3 m}$ and $J_{5 m}$ and $J_{6 m}$ in (24).

It then remains to calculate $J_{2 m}$ and $J_{4 m}$. Their integrands become highly oscillatory when $\theta \rightarrow \pi / 2$. Following Xie et al. (2005):

$u=|\gamma| \frac{\sin \theta}{\cos ^{2} \theta}$

and where $\gamma=(y-b) / 2 a F_{n}^{2}(m=1,4,6,7)$, or $\gamma=(x-a) / 2 a F_{n}^{2}$ ( $m=2,3,5,8)$, where $F_{n}=U / \sqrt{2 a g}$ is the Froude number for the element. Thus, the oscillatory behaviour is 'stretched':

$k_{2} s_{1}=\psi(\theta)\left[\frac{\sqrt{2 \alpha u}}{\sqrt{|\gamma| \sqrt{4 u^{2}+\gamma^{2}-\gamma^{2}}}}+\frac{\gamma}{|\gamma|} u\right]$

where $\alpha=(x-a) / 2 a F_{n}^{2}, \psi(\theta)=0.5(1+2 \tau \cos \theta+\sqrt{1+4 \tau \cos \theta}$.

Care is also advised when handling the logarithmic singularities in the free-surface elevation integrals (25)-(31).

\section{Numerical results}

A rectangular pressure patch is selected for validation of the numerical schemes outlined in Section 3. The length-beam ratio of the pressure patch is $a / b=1$ and patch length Froude number $F_{n}=0.50$. The free-surface elevations of the rectangular pressure patch at reduced frequencies of $\tau=0.2375$ and 0.275 (below and above the critical value of 0.25 ) are compared with existing results. Due to the phase effect, there are real (Re) and imaginary (Im) parts for the free-surface elevation. The same applies to the escape area and escape volume. Wave-cuts at $y=b$ were compared with those of Doctors (1976), who used a different numerical scheme. The agreement was good (see Figs. 3 and 4).

The non-dimensional free-surface elevation per unit pressure is defined as:

$\tilde{\varsigma}_{\mathrm{p}}=\rho_{\mathrm{w}} g \varsigma_{\mathrm{p}}$.

The corresponding non-dimensional escape area and escape volume per unit pressure are

$\tilde{\alpha}=\frac{\rho_{\mathrm{w}} g \alpha}{L}$

$\tilde{v}_{\mathrm{p}}=\frac{\rho_{\mathrm{w}} g v_{\mathrm{p}}}{L^{2}}$

Numerical predictions were also carried out for an ALV having principal dimensions as given in Table 1 .

The cushion area of the ALV is divided into a number of rectangular elements (see Fig. 2). Some convergence studies have been carried out to find the proper discretisation scheme. Figs. 5 and 6 show real and imaginary part of the non-dimensional free-surface elevation at the cushion longitudinal outer boundary when $F_{n}=0.5$, respectively. The free-surface elevation is smooth both in space and reduced frequency due to proper handling of the singularities and the high oscillating behaviour. It can be also observed that the free-surface decrease as the reduced

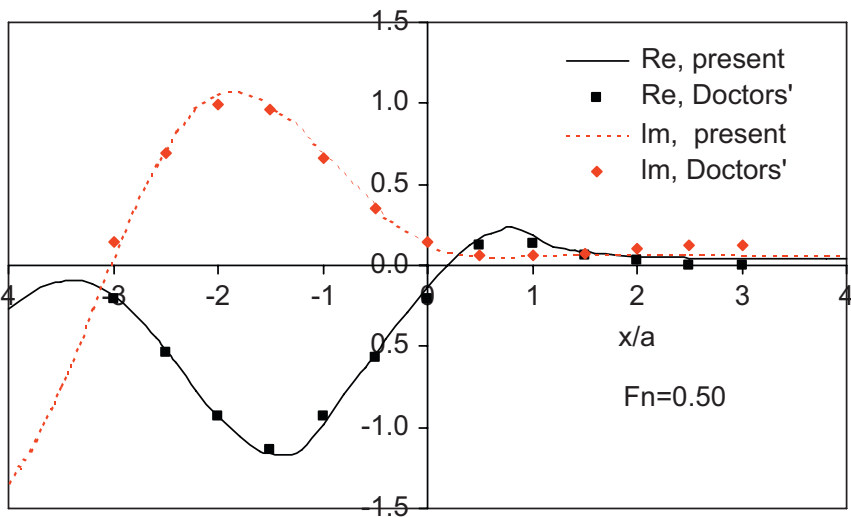

Fig. 3. Non-dimensional free-surface elevation $\tilde{\varsigma}_{\mathrm{p}} y=b$ for a rectangular pressure patch, $b / a=1, \tau=0.2375$

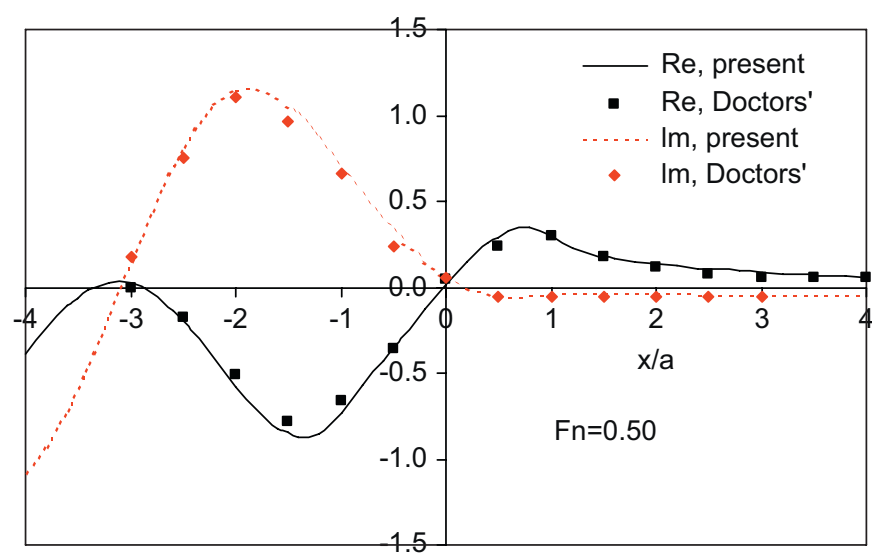

Fig. 4. Non-dimensional free-surface elevation $\tilde{\varsigma}_{\mathrm{p}} y=b$ for a rectangular pressure patch, $b / a=1, \tau=0.275$.

Table 1

Principal dimensions of the ALV

\begin{tabular}{lc}
\hline Length, $L_{\mathrm{OA}}(\mathrm{m})$ & 40.0 \\
Displacement, $\Delta\left(\mathrm{m}^{3}\right)$ & 170 \\
Breadth, $B(\mathrm{~m})$ & 15.0 \\
Separation, $R(\mathrm{~m})$ & 9.60
\end{tabular}

frequency, $\tau$, increases, which mean that at high (reduced) frequencies, the free-surface elevation responses much less. Figs. 7 and 8 show non-dimensional free-surface elevations at the stern of the ALV when $F_{n}=1.0$. The calculated nondimensional escape areas at the stern opening of the ALV for Froude numbers $F_{n}=0.5,1.0,1.5$ are shown in Figs. 9-11, respectively. Both demi-hull and twin-hull results are given. In the twin-hull calculation cases, the effect of pressure of one demi-hull on the free-surface elevation of another demi-hull is taken into consideration. It can be seen that the demi-hull has some effect on the escape area of the other demi-hull only for $F_{n}=0.5$ and at lower values of frequency. With vessel speed increase, this effect become much less and may be neglected. The escape areas decay quickly with increasing encounter frequency. Also the magnitude of the escape area at lower Froude number is larger than that at higher Froude numbers. Since the craft is normally operating at high speed, this may be probably the reason the craft has good seakeeping 


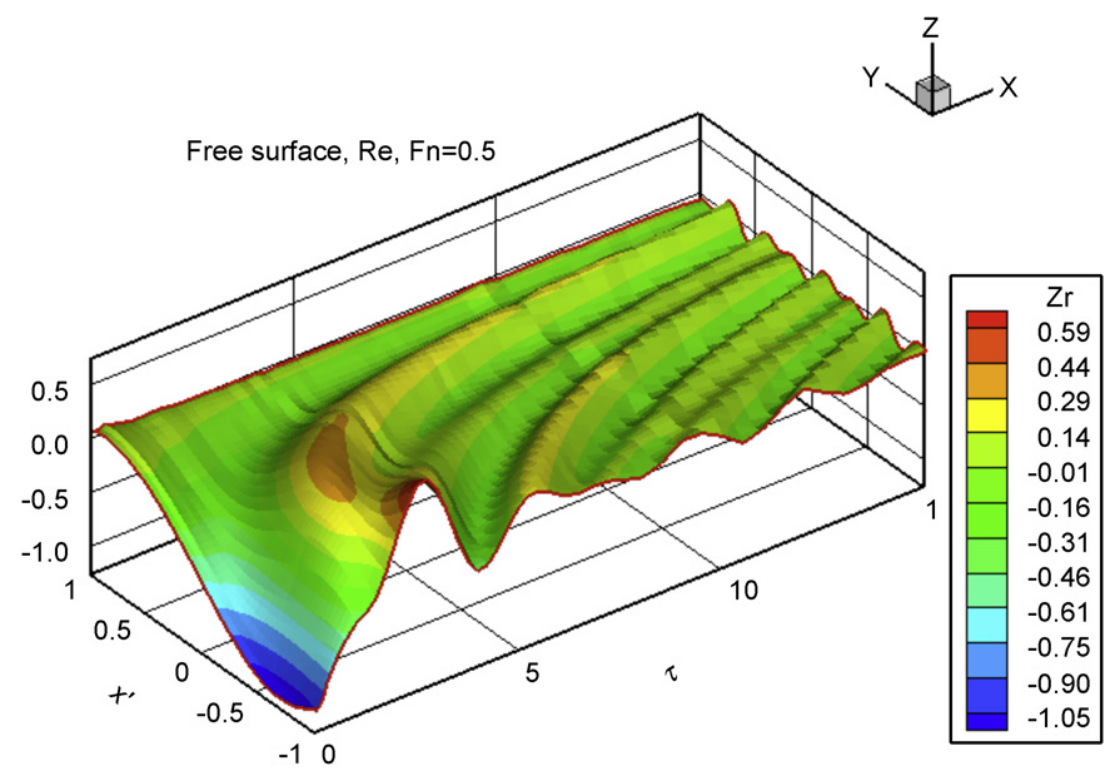

Fig. 5. Real part of $\tilde{\varsigma}_{p}$ at cushion outer boundary of the ALV.

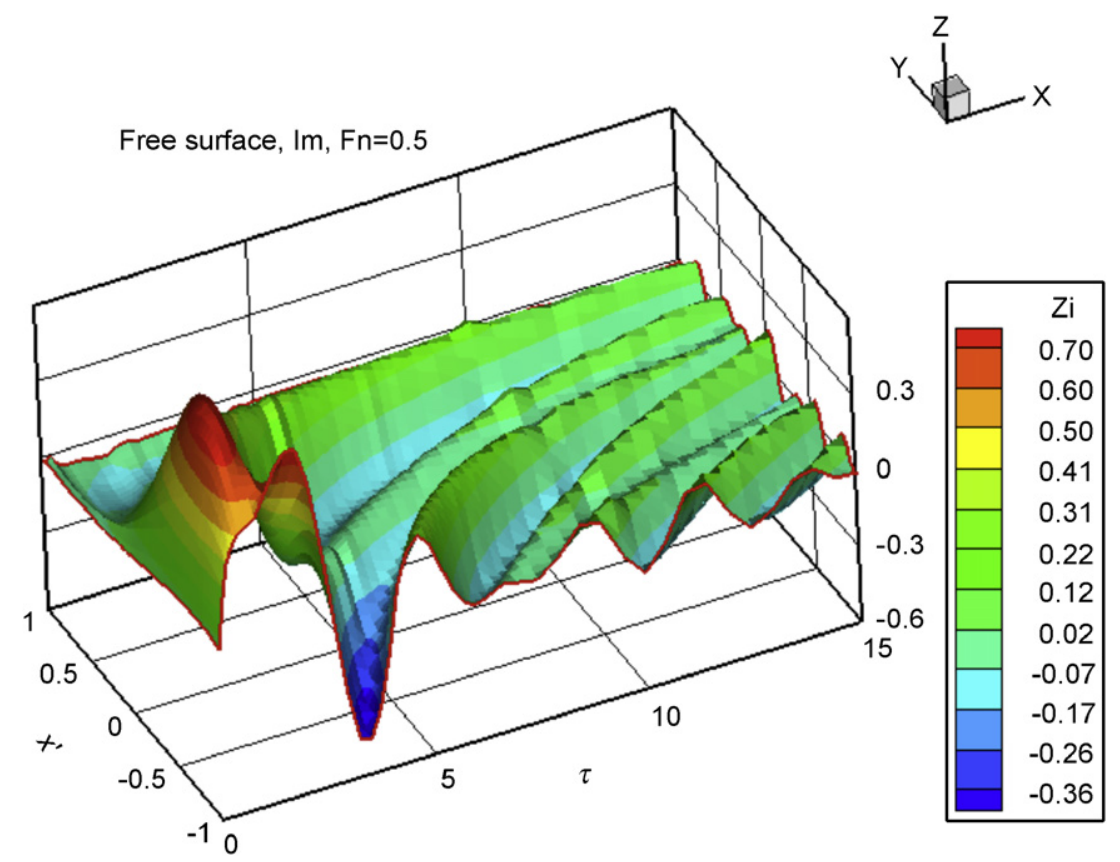

Fig. 6. Imaginary part of $\tilde{\varsigma}_{\mathrm{p}}$ at cushion outer boundary of the ALV.

performance. The cushion escape volumes of the ALV for Froude numbers $F_{n}=0.5,1.0,2.0$ are shown in Figs. 12-14, respectively. The trends are similar to that of the escape area, there will be less free-surface disturbance inside the cushion of the ALV for higher vessel speed.

Using the computed values (46) and (47) in (6) and (20) yields the transfer functions for heave, pitch and roll, from which the spectral responses may be obtained. The RMS of heave, pitch and vertical acceleration on bow of the ALV at $F_{n}=1.0$ and four mean wave periods $T_{01}=4,6,8$ and $10 \mathrm{~s}$ are plotted in Fig. 15-17, respectively, for all heading angles at interval of $30^{\circ}$. Generally speaking, there will be less craft responses for shorter waves (smaller mean wave periods). Also, the vehicle has less response to head wave than to following seas, this may be due to the characteristics of the free-surface elevation, escape area and escape volume of the cushion of the ALV. At high values of the reduced frequency, the escape area and escape volume both tend to zero: the vessel rides the waves. Figs. 18 and 19 show heave and vertical acceleration at bow of the ALV at $F_{n}=1.5$, respectively. Again, the vertical acceleration on bow at following waves is much larger than that at head waves. Although there is no experimental results available for validation of the approach at this stage, the results obtained by the present approach seems reasonable. 


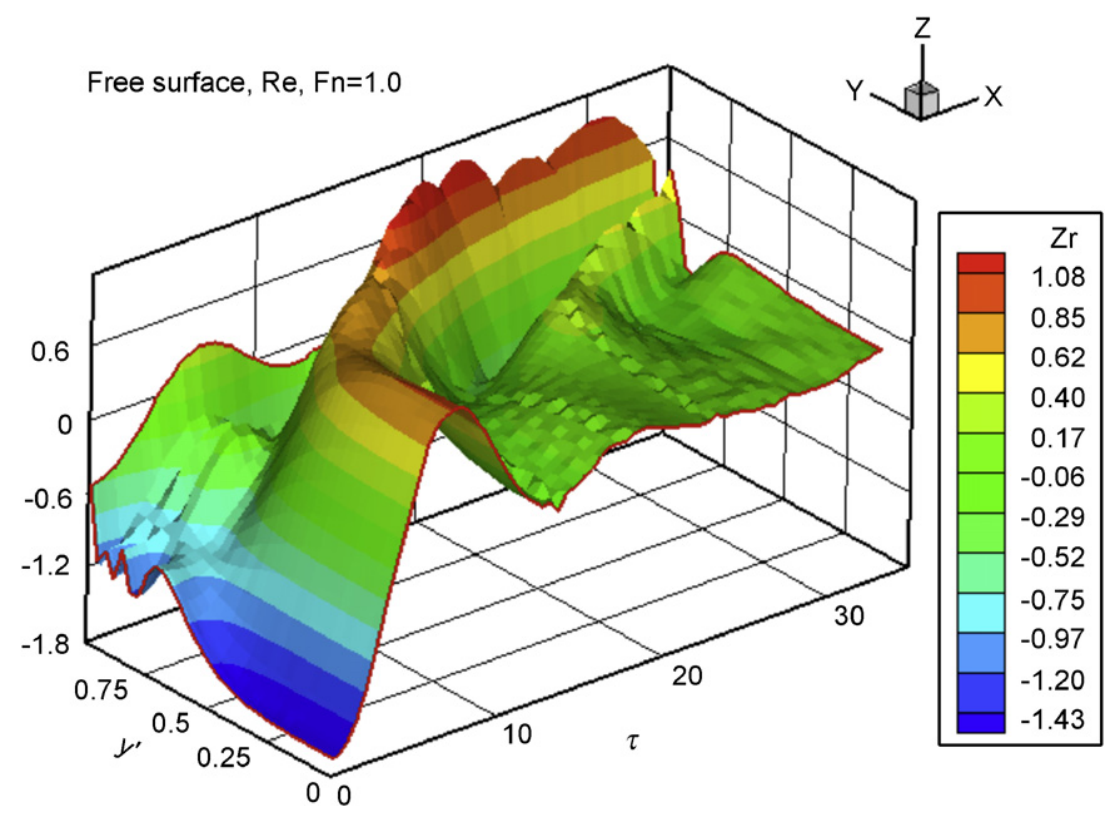

Fig. 7. Real part of $\tilde{\varsigma}_{\mathrm{p}}$ at cushion stern boundary of the ALV.

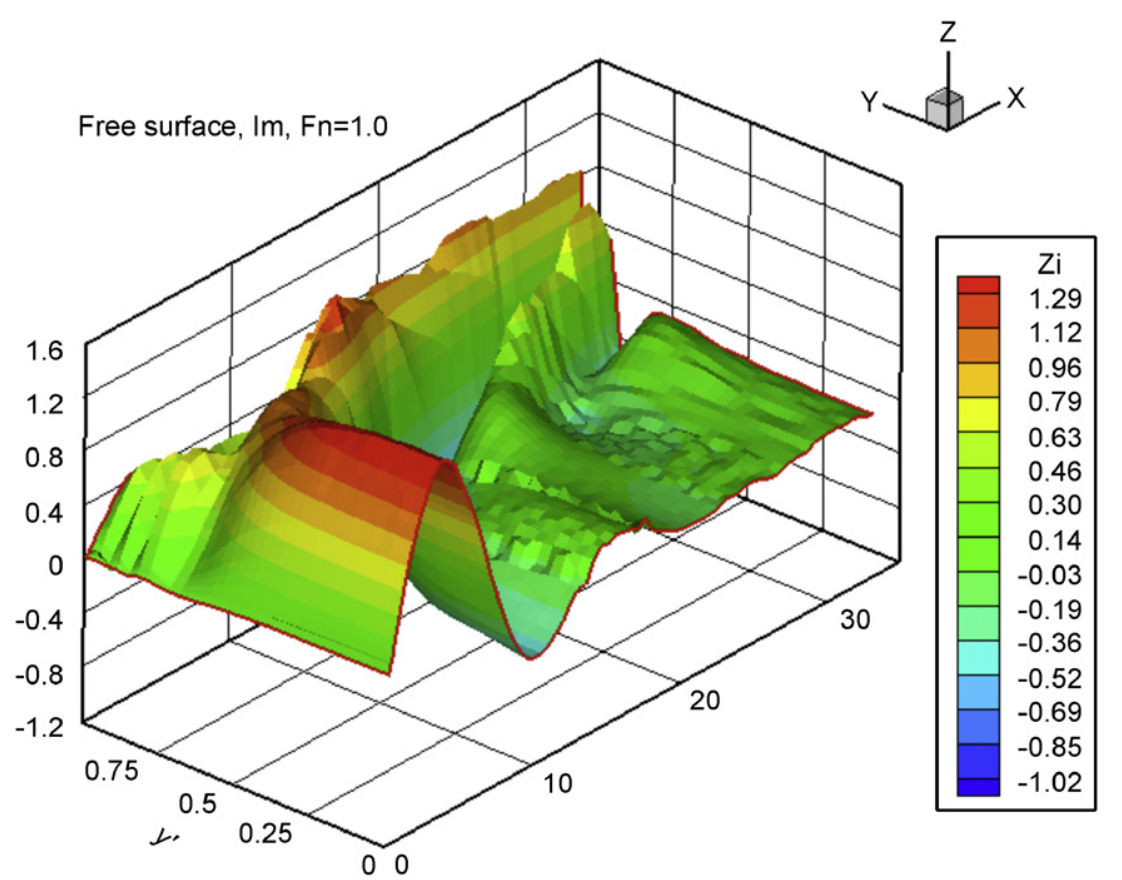

Fig. 8. Imaginary part of $\tilde{\varsigma}_{\mathrm{p}}$ at cushion stern boundary of the ALV.

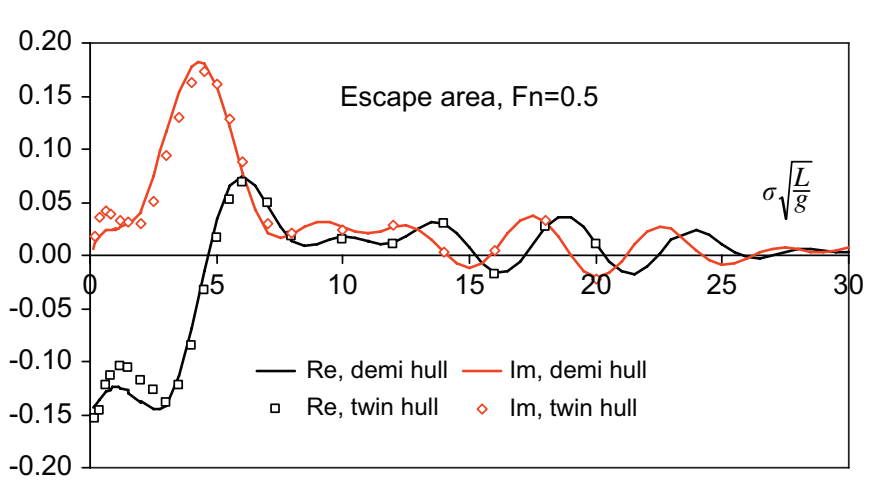

Fig. 9. Non-dimensional escape area $\tilde{\alpha}_{\mathrm{p}}$ at stern of the ALV.

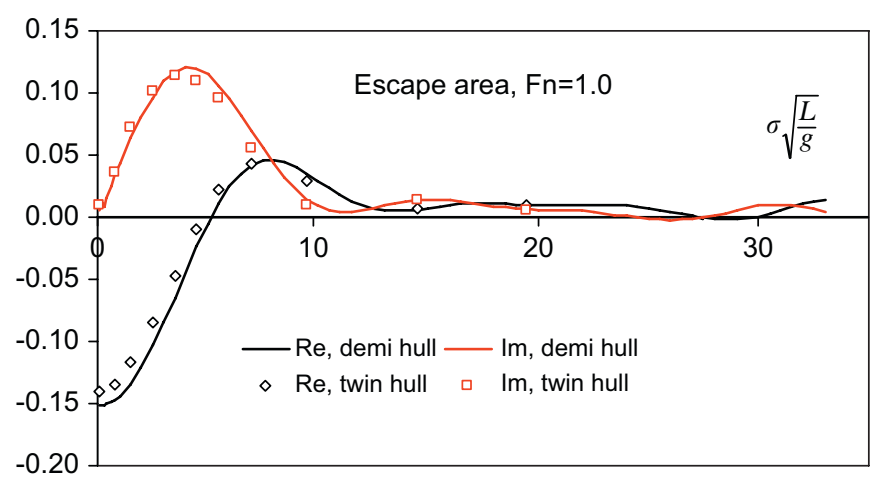

Fig. 10. Non-dimensional escape area $\tilde{\alpha}_{\mathrm{p}}$ at stern of the ALV. 


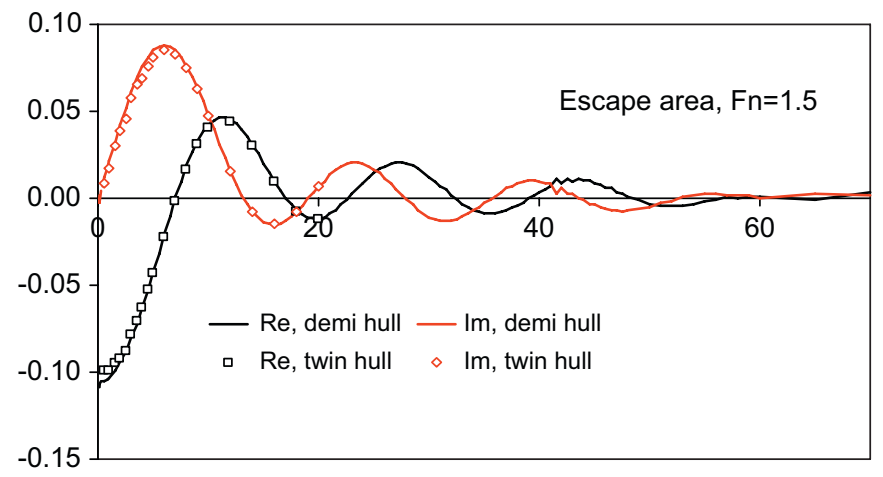

Fig. 11. Non-dimensional escape area $\tilde{\alpha}_{p}$ at stern of the ALV.

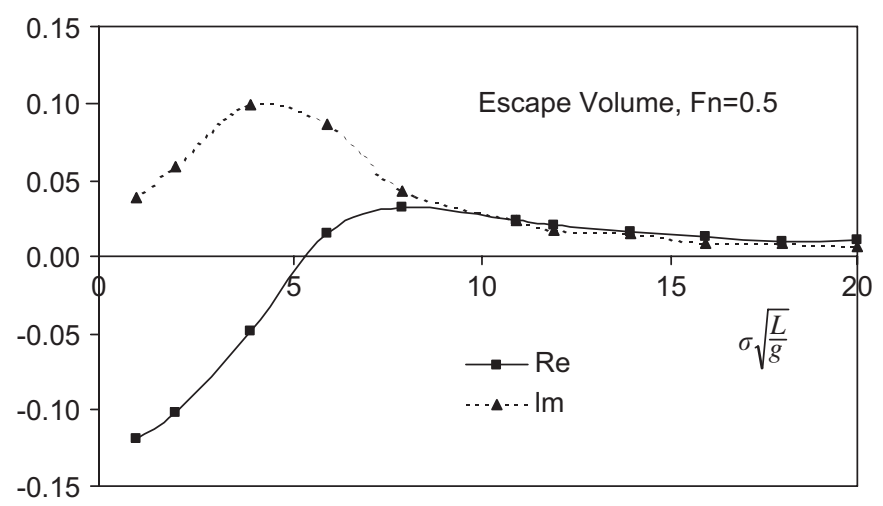

Fig. 12. Non-dimensional escape volume $\tilde{v}_{\mathrm{p}}$ of the ALV.

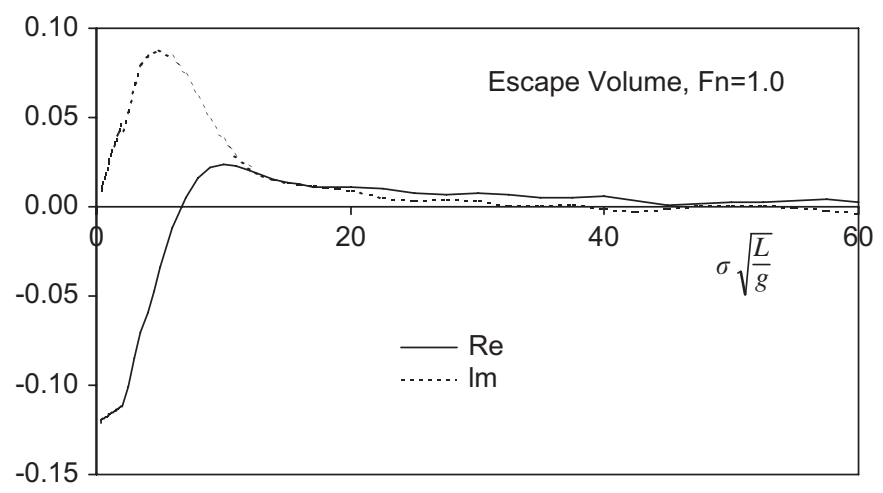

Fig. 13. Non-dimensional escape volume $\tilde{v}_{\mathrm{p}}$ of the ALV.

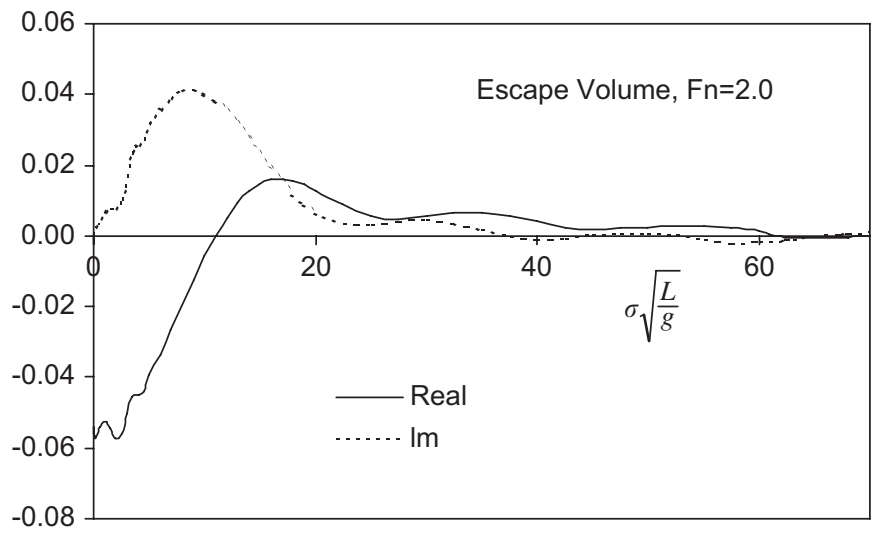

Fig. 14. Non-dimensional escape volume $\tilde{v}_{\mathrm{p}}$ of the ALV.

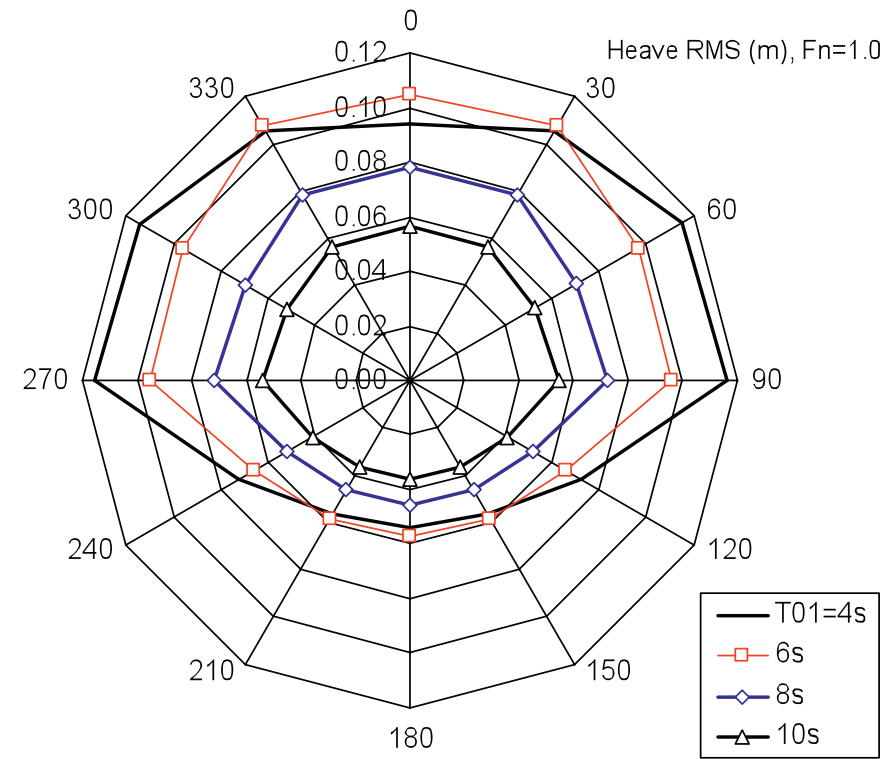

Fig. 15. RMS of heave motion of the ALV at $F_{n}=1.0$ for $H_{1 / 3}=1 \mathrm{~m}$.

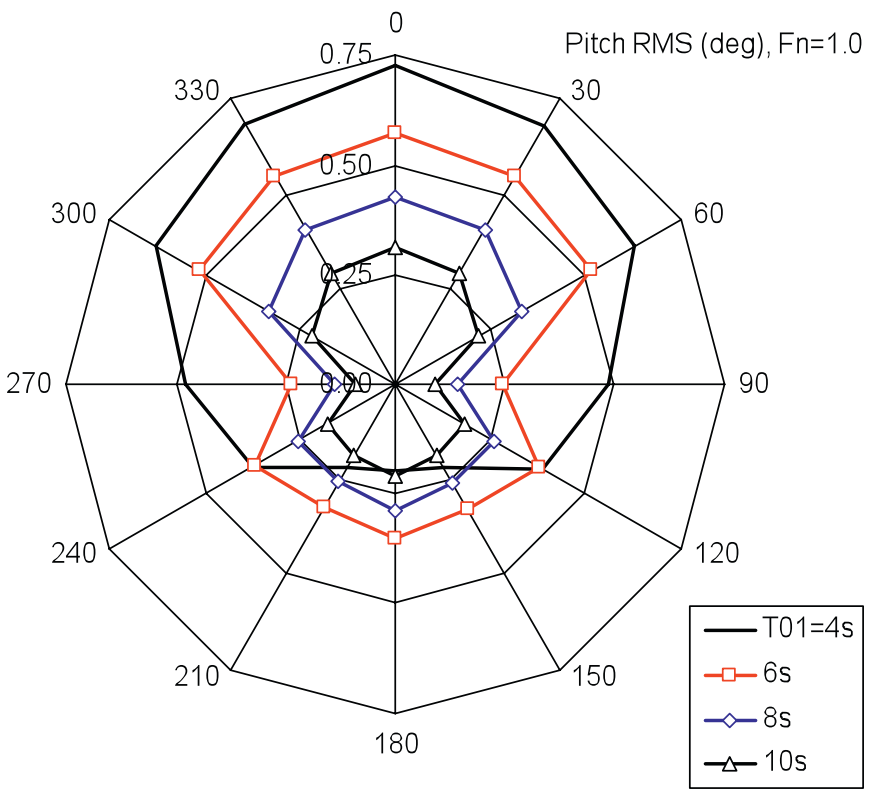

Fig. 16. RMS of pitch motion of the ALV at $F_{n}=1.0$ for $H_{1 / 3}=1 \mathrm{~m}$.

\section{Conclusions}

A mathematical model has been developed to predict the seakeeping performance of an ALV in the frequency domain. The external forces/moments acting on the hull arise from hydrodynamic (added mass and damping) loads, wave excitation-calculated by strip theory-and cushion pressure effects. Attention has focused on the latter: the present method is based on a moving, oscillatory pressure patch. A efficient and effective numerical scheme has been proposed to handle the singularities and highly oscillatory behaviour of several integrands. Results are in good agreement with available published data for the freesurface elevation in the cushion. Numerical results for the escape area, escape volume, and for the vessel motions of the ALV are 
0 RMS of Vertical acc. at bow (g), $F n=1.0$

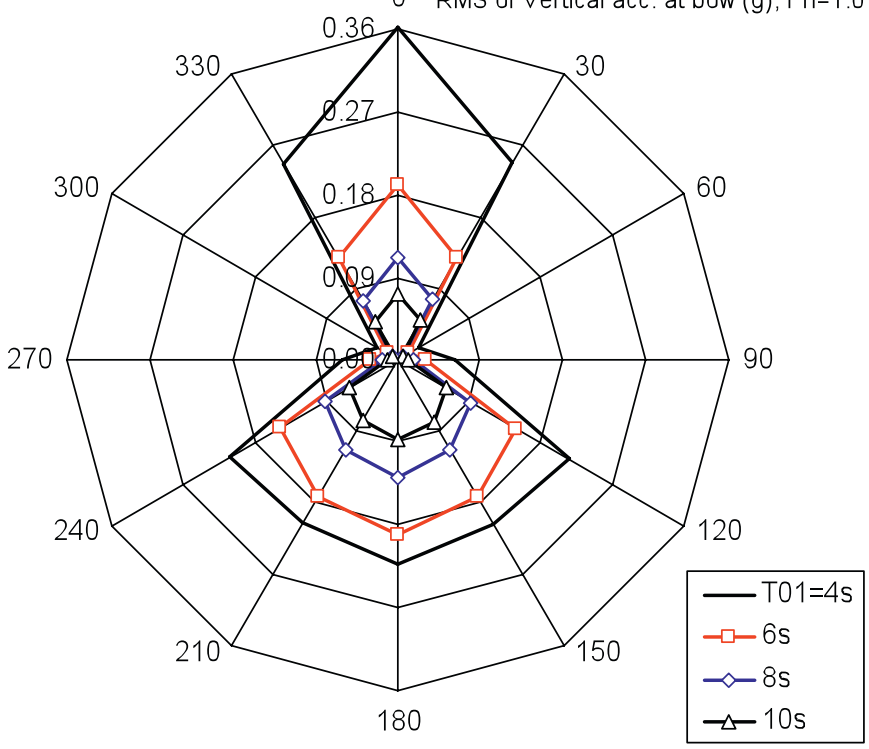

Fig. 17. RMS of vertical acceleration at bow of the ALV at $F_{n}=1.0$ for $H_{1 / 3}=1 \mathrm{~m}$.

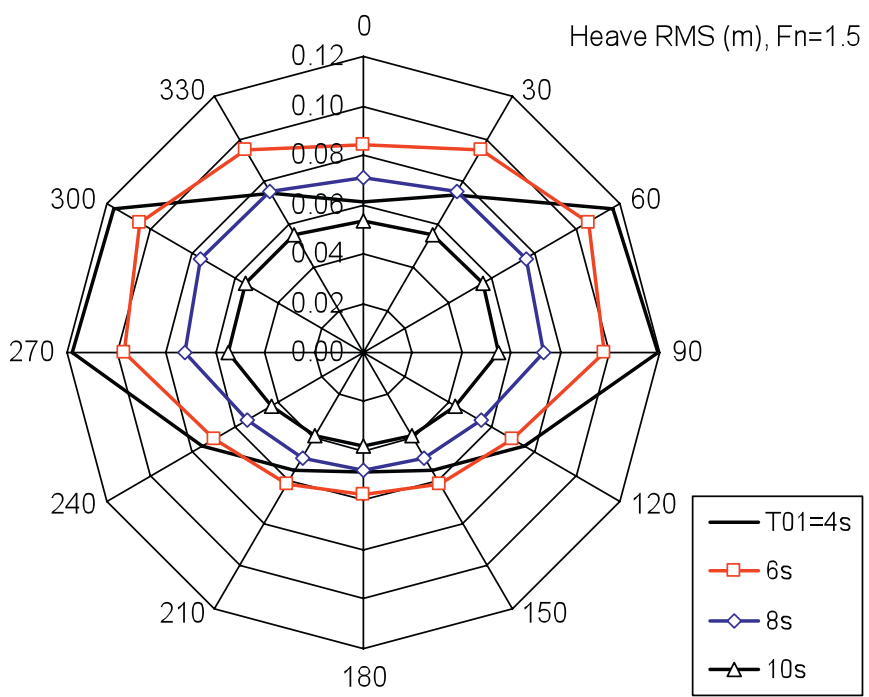

Fig. 18. RMS of heave motion of the ALV at $F_{n}=1.5$ for $H_{1 / 3}=1 \mathrm{~m}$.

provided and discussed. The present method therefore appears to provide a helpful means of assessing performance at both design and operational stages.

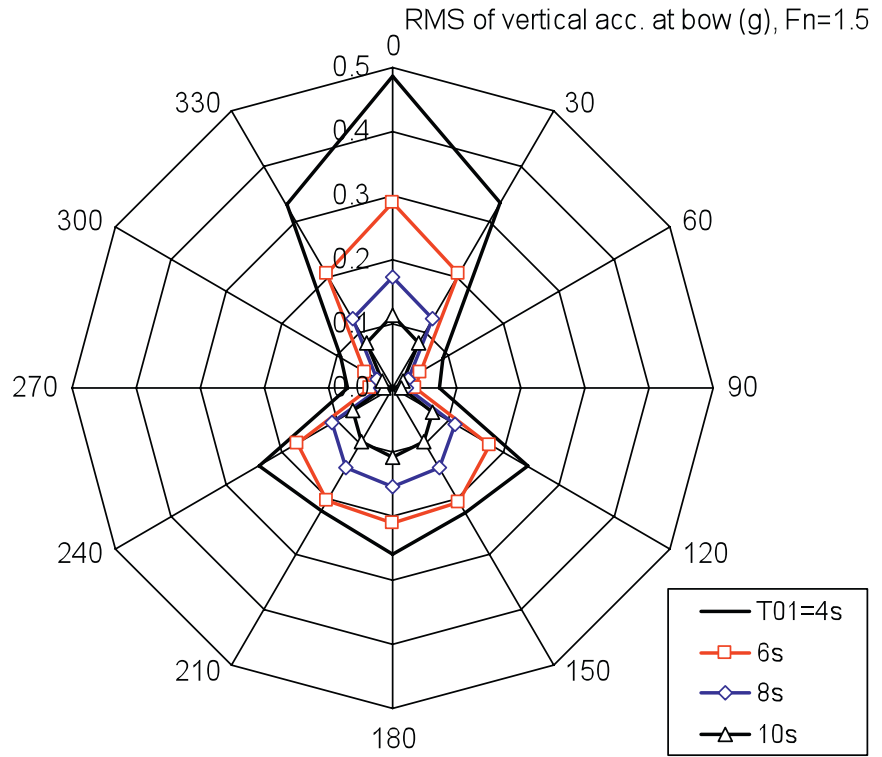

Fig. 19. RMS of vertical acceleration at bow of the ALV at $F_{n}=1.5$ for $H_{1 / 3}=1 \mathrm{~m}$.

\section{References}

Abramowitz, M., Stegun, I.A., 1970. Handbook of Mathematical Functions. Dover Publications Inc., New York.

Allenstrom, B, Liljenberg, H., Tuden, U., 2001. An Air lifted catamaran. In: Proceedings of the FAST 2001, Southampton, UK.

Allenstrom, B., Liljenberg, H., Tuden, U., 2003. Concept development and model testing - new generation air assisted vessels (AVV) with water jet propulsion. In: Proceedings of the FAST 2003, Naples, Italy.

Chen, H.H., 1977. On a rectangular pressure distribution of oscillating strength moving over a free surface. Journal of Ship Research 21 (1), 11-23.

Doctors, L.J., 1976. The effect of air compressibility on the nonlinear motion of an air-cushion vehicle over waves. In: Proceedings of the 11th ONR Symposium on Naval Hydrodynamics, UCL, London, pp. 373-388.

EFFISES Project, 2001-2004. Energy Efficient Safe Innovative Ships and Vehicle, European Commission CEC Contract no. GRD1-2000-25847, coordinated by SES Europe.

Faltinsen, O.M., 2005. Hydrodynamics of High-Speed Marine Vehicles. Cambridge University Press.

Kaplan, P., Bentson, J., Davis, S., 1981. Dynamics and hydrodynamics of surface effect ships. Transactions of the SNAME 89, 211-247.

Kim, C.H., Tsakonas, S., 1981. An analysis of heave added mass and damping of a surface effect ship. Journal of Ship Research 25 (1).

Wehausen, J.V., Laitone, E.V., 1960. Surface waves in fluid dynamics III. In: Flugge, S., Truesdell, C. (Eds.), Handbuch der Physik, vol. 9. Springer, Berlin, pp. 446-778 (chapter 3).

Xie, N., Vassalos, D., Jasionowski, A., 2005. A study of the hydrodynamics of a threedimensional planing surface. Ocean Engineering 32, 1539-1555. 